\title{
RECENT RESULTS ON LIGHT HADRON SPECTROSCOPY AT BESIII
}

\author{
Y. CHEN \\ (for the BESIII Collaboration) \\ Institute of High Energy Physics, Academy of Science, \\ Beijing 100049, P. R. China \\ chenye@ihep.ac.cn
}

\begin{abstract}
Several measurements on light hadron spectroscopy have been achieved with Beijing Spectrometry III (BESIII). BESII results such as a near threshold enhancement on the $p \bar{p}$ invariants mass spectrum namely $\mathrm{X}(1860)$ and a resonance $\mathrm{X}(1835)$ have been confirmed with 225 million $J / \psi$ data accumulated from June 12, 2009 to July 28, 2009. Along with some other preliminary BESIII results including observations of X(1870), X(2120) and $\mathrm{X}(2370)$; the first direct measurement of $a_{0}(980) / f_{0}(980)$ mixing; and an improved measurement on the matrix element of decay $\eta^{\prime} \rightarrow \pi \pi \eta$ have been reported at the 4 th international workshop on charm physics.
\end{abstract}

Keywords: Light hadron spectroscopy; $J / \psi$ decay

PACS numbers: 13.20Gd, 14.40Be, 13.75Cs, $12.39 \mathrm{Mk}$

\section{Introduction}

Study of the spectroscopy is a way to get a better understanding of the internal structure. We choose to study the $\tau-$ charm collider because $e^{+} e^{-}$collider is a gluon rich process and it could provide an environment with relatively low background. Using the 225 million $J / \psi$ data sample accumulated at BESIII, we are dedicated to make more precise measurements on the established spectrum of the light hadrons, search for the non-conventional hadrons, understand how hadrons are formed ${ }^{1}$. This talk presents five decay channel analysis performed at BESIII which would be described in the following sections.

\section{2. $p \bar{p}$ invariant mass spectrum enhancement}

An anomalous near threshold enhancement in $p \bar{p}$ invariant mass spectrum, $\mathrm{X}(1860)$, has been observed at $\mathrm{BESII}^{2}$ and recently confirmed by BESIII ${ }^{3}$. The mass of this particle is measured to be $1.859_{-10}^{+3}$ (stat. $)_{-25}^{+5}$ (syst.) $\mathrm{GeV}$ and the total width is $\Gamma<30 \mathrm{MeV} / \mathrm{c}^{2}$ at the $90 \%$ confidence level $^{2}$. This enhancement is recently confirmed again by CLEO collaboration ${ }^{4}$. 
The discovery of $\mathrm{X}(1860)$ invokes intensive studies on light hadron spectroscopes both experimentally ${ }^{5,6,7,8}$ and phenomenologically ${ }^{9,10,11,12,13,14,15}$. Experiment results shows that this enhancement is not seen from other quarkonia decay such as $J / \psi \rightarrow \pi^{0} p \bar{p}^{2}, \Upsilon(2 S) \rightarrow \gamma p \bar{p}^{5}, \psi \rightarrow \gamma p \bar{p}^{6}$ and $J / \psi \rightarrow \omega p \bar{p}^{7}$. Phenomenologically, people interpret the originality of $\mathrm{X}(1860)$ as baryonium, multiquarks candidate or mainly from pure final state interaction etc. Meanwhile, people intent to associate this enhancement with a broad enhancement observed from B meson decays ${ }^{16,17}$ or a new resonance $\mathrm{X}(1835)$ observed at $\mathrm{BESII}^{18}$. BESIII study the $p \bar{p}$ invariant mass spectrum using both the decay channel of $\psi \rightarrow \pi \pi J / \psi, J / \psi \rightarrow \gamma p \bar{p}^{3}$ and also $J / \psi \rightarrow \gamma p \bar{p}$. From the left plot of Fig. 1, we see the the structure of $\mathrm{X}(1860)$ has been clearly confirmed.

\section{Confirmation of $\mathrm{X}(\mathbf{1 8 3 5})$ and observation of two new structures in $J / \psi \rightarrow \gamma \pi \pi \eta^{\prime}$}

$\mathrm{X}(1835)$ was observed at BESII in the decay of $J / \psi \rightarrow \gamma \pi \pi \eta^{\prime}$, the mass is measured to be $1833.7 \pm 6.1$ (stat.) \pm 2.7 (syst.) $\mathrm{MeV}$ and the width is measured to be $67.7 \pm 20.3$ (stat) \pm 7.7 (stat) $\mathrm{MeV}^{18}$. Confirmation of $\mathrm{X}(1835)$ is straightforward and necessary at BSEIII; other motivation of this study would be that LQCD predicts the $0^{-+}$glueball mass is around $2.3 \mathrm{GeV}$. It would be important and interesting to study the $J / \psi \rightarrow \gamma \pi \pi \eta^{\prime}$. The right plot of Fig 1 shows the invariant mass spectrum of $J / \psi \rightarrow \gamma \pi \pi \eta^{\prime}$ with two $\eta^{\prime}$ decay modes $\eta^{\prime} \rightarrow \gamma \rho$ and $\eta^{\prime} \rightarrow \pi \pi \eta$. We see $X(1835)$ and $\eta_{c}$ are observed along with two additional structures around $2.1 \mathrm{GeV}$ and $2.3 \mathrm{GeV}$. Signal of $f_{1}(1510)$ is also clearly seen as well ${ }^{19}$.

From above, we conclude that $\mathrm{X}(1835)$ resonance is confirmed at BESIII with a significance of $20 \sigma$. The width is significantly larger than that measured at BESII with one resonance in the fit. Meanwhile, X(2120) and X(2370), are observed with significance larger than $7.2 \sigma$ and $6.4 \sigma$, respectively ${ }^{19}$.
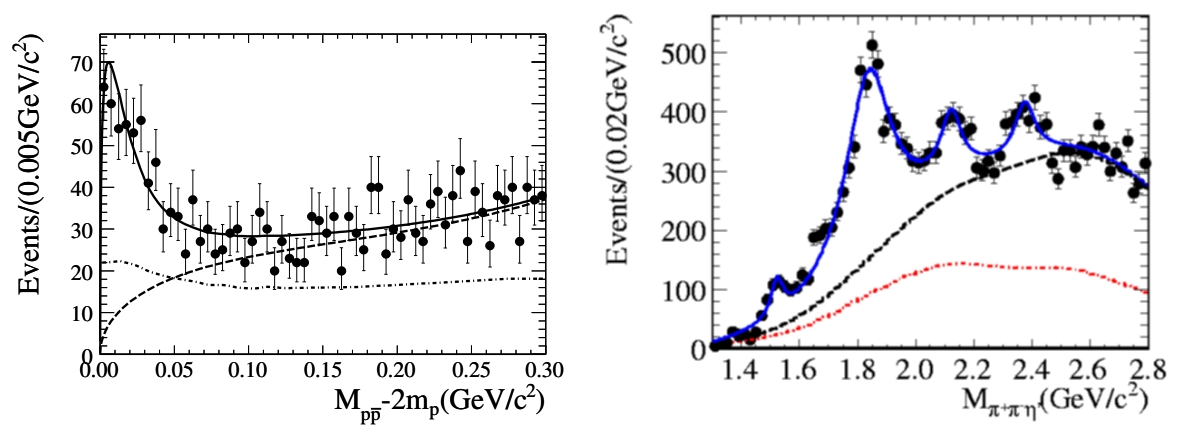

Fig. 1. Left: $p \bar{p}$ invariant mass spectrum from the $\psi^{\prime} \rightarrow \pi \pi J / \psi(J / \psi \rightarrow \gamma p \bar{p})$, solid curve is the fitting result, the dashed curve is the background, the dash-dotted line is the efficiency curve; Right: invariant mass spectrum of $\pi \pi \eta^{\prime}$ fitting with four resonances model. the red dash-dot line is contribution of non- $\eta^{\prime}$ events and the $\pi^{0} \pi \pi \eta^{\prime}$ background for two $\eta^{\prime}$ decay modes and the solid black line is including an additional empirical function to describe the phase-space contribution. 


\section{Observation of $X(\mathbf{1 8 7 0}) \rightarrow a_{0} \pi$ in $J / \psi \rightarrow \omega \pi \pi \eta$ at BESIII.}

We also study the decay channel of $J / \psi \rightarrow \omega \pi \pi \eta$. Fig. 2 demonstrates a fit with three resonances model. Study shows that the $f_{1}(1285), \eta(1410)$ and $\mathrm{X}(1870)$ are primarily decay via $a_{0}(980) \pi^{ \pm}$. Whether the $\mathrm{X}(1870)$ is the $\mathrm{X}(1835), \eta_{2}(1870)$ or a new resonance, further study is needed.

\section{5. $a_{0}(980)-f_{0}(980)$ mixing}

There are long-history controversial views on the nature of $a_{0}(980) / f_{0}(980)$. People interpret them as $q \bar{q}$ measons, four quarks states, hybrids and $K \bar{K}$ molecules etc. Study of the mixing of $a_{0}(980)$ and $f_{0}(980)$ will shed new light on this mystery. A narrow peak has been predicted around $8 \mathrm{MeV}$ between the charged and neutral kaon thresholds $(987 \sim 995 \mathrm{MeV})^{21,22,23}$ which motives us to do this study.

A direct measurement of the $a_{0}(980)-f_{0}(980)$ mixing has been firstly carried out at BESIII. The $f_{0}(980)$ to $a_{0}(980)$ transition is measured via $J / \psi \rightarrow \phi f_{0}(980) \rightarrow$ $\phi a_{0}(980) \rightarrow \phi \eta \pi^{0}$. Evidence for $f_{0}(980) \rightarrow a_{0}(980)$ is found with a significance of $3.4 \sigma$, see the left plot of Fig. 3. For the case of $a_{0}^{0}(980) \rightarrow f_{0}(980)$ transition, it is measured in the processes $\chi_{c 1} \rightarrow \pi^{0} a_{0}^{0}(980) \rightarrow \pi^{0} f_{0}(980) \rightarrow \pi^{0} \pi \pi$, and the significance is $1.9 \sigma$, shown on the right plot of Fig. 3. Measurements and upper limits of both branching ratios and mixing intensities are determined as well ${ }^{20}$. Following the mixing intensity of $\xi_{f a}$ and $\xi_{a f}$ is presented respectively,

$$
\begin{aligned}
& \xi_{f a}=\frac{B r\left(J / \psi \rightarrow \phi f_{0}(980) \rightarrow \phi a_{0}(980) \rightarrow \phi \eta \pi^{0}\right)}{B r\left(J / \psi \rightarrow \phi f_{0}(980) \rightarrow \phi \pi \pi\right)} \\
= & (0.60 \pm 0.20(\text { stat. }) \pm 0.12(\text { sys. }) \pm 0.26(\text { para. })) \%
\end{aligned}
$$

and

$$
\begin{aligned}
& \xi_{a f}=\frac{B r\left(\chi_{c 1} \rightarrow \pi^{0} a_{0}(980) \rightarrow \pi^{0} f_{0}(980) \rightarrow \pi^{0} \pi \pi\right)}{B r\left(\chi_{c 1} \rightarrow \pi^{0} a_{0}(980) \rightarrow \pi^{0} \pi^{0} \eta\right)} \\
& =(0.31 \pm 0.16(\text { stat. }) \pm 0.14(\text { sys. }) \pm 0.03(\text { para. })) \% .
\end{aligned}
$$

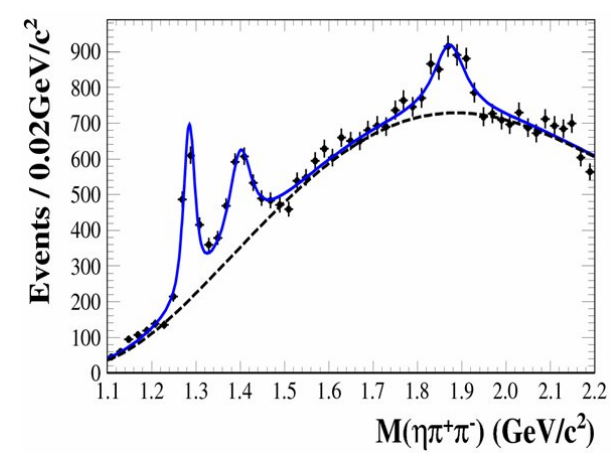

Fig. 2. $J / \psi \rightarrow \omega \pi \pi \eta . \pi \pi \eta$ invariant mass spectrum with three resonances model, the dashed line is the background component described by polynomial function. 

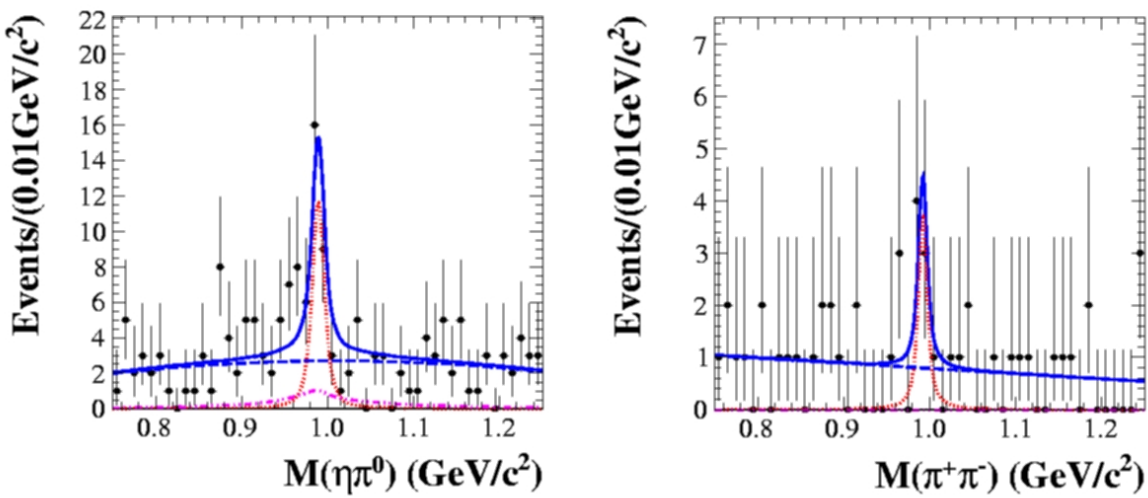

Fig. 3. (a)Fitting result of the $\eta \pi^{0}$ mass spectrum recoiling against the $\phi$ signal. (b) Fitting result of the $\pi^{+} \pi^{-}$mass spectrum in the $\chi_{c 1}$ mass window. The dotted lines shows the mixing signal. The dash-dotted lines indicate underlying $a_{0}(980)$ of $f_{0}(980)$ from other processes. The dashed lines denote the polynomial background.

Table. I Experimental and theoretical values of the parameters of the matrix element squared for $\eta^{\prime} \rightarrow \pi \pi \eta$.

\begin{tabular}{|l|c|c|c|}
\hline Par. & VES $^{25}$ & Theory $^{27}$ & BESIII $^{24}$ \\
$\mathrm{a}$ & $-0.127 \pm 0.018$ & $-0.116 \pm 0.001$ & $-0.047 \pm 0.012$ \\
$\mathrm{~b}$ & $-0.106 \pm 0.032$ & $-0.042 \pm 0.034$ & $-0.069 \pm 0.021$ \\
$\mathrm{c}$ & $0.015 \pm 0.018$ & - & $0.019 \pm 0.012$ \\
$\mathrm{~d}$ & $-0.082 \pm 0.019$ & $0.010 \pm 0.019$ & $-0.073 \pm 0.013$ \\
\hline \hline Par. & CLEO $^{26}$ & VES $^{28}$ & BESIII $^{24}$ \\
$\operatorname{Re}(\alpha)$ & $-0.021 \pm 0.025$ & $-0.072 \pm 0.014$ & $-0.033 \pm 0.006$ \\
$\operatorname{Im}(\alpha)$ & 0.000 (fixed) & $0.000 \pm 0.100$ & $0.000 \pm 0.050$ \\
$\mathrm{c}$ & 0.000 (fixed) & $0.020 \pm 0.019$ & $0.018 \pm 0.010$ \\
$\mathrm{~d}$ & 0.000 (fixed) & $-0.066 \pm 0.034$ & $-0.059 \pm 0.013$ \\
\hline
\end{tabular}

\section{Matrix element measurement of the decay $\eta^{\prime} \rightarrow \eta \pi \pi$}

A new level of precision measurement on the matrix element for the decay of $\eta^{\prime} \rightarrow \eta \pi \pi$ has also been performed based on the largest sample of $\eta^{\prime}$ decay to data. Two representations of parametrization for the decay amplitudes are adopted including the general decomposition and the linear decomposition. Table. I demonstrates the experimental and theoretical values of the parameters of the matrix 
element squared for $\eta^{\prime} \rightarrow \pi \pi \eta$ in the general parametrization (upper) and in the linear parametrization (lower) ${ }^{24}$.

\section{Acknowledgments}

The speaker would like to thank all the BESIII colleagues for their contribution to make this talk possible. And many thanks to the organizers of Charm2010.

\section{References}

1. "Physics at BESIII", edited by K. T. Chao and Y. F. Wang, Int. J. Mod. Phys. A 24, suppl. 1 (2009).

2. J.Z. Bai et al. (BES Collaboration), Phys. Rev. Lett. 91, 022001 (2003).

3. M. Ablikim et al. (BESIII Collaboration), Chinese Physics C 34, 4 (2010).

4. J. P. Alexander et al. (CLEO Collaboration), Phys. Rev. D82, 092002 (2010).

5. S. A. Athar et al. (CLEO Collaboration), Phys. Rev. D, 032001 (2006).

6. M. Ablikim et al. (BES Collaboration), Phys. Rev. Lett. 99, 011802 (2007).

7. M. Ablikim et al. (BES Collaboration), Eur. Phys. J. C 53, 15 (2008).

8. For review, S. Jin, Int. J. Mod. Phys. A 20, 5145 (2005).

9. A. Datta and P. J. O'Donnel, Phys. Lett. B567, 273 (2003).

10. M. L. Yan, S. Li, B. Wu, B. Q. Ma, Phys. Rev. D 72, 034027 (2005).

11. S. L. Zhu, Int. J. Mod. Phys. A20,1548 (2005).

12. B. S. Zou, H. C. Chiang, Phys. Rev. D 69, 034004 (2004).

13. D. V. Bugg, Phys. Lett. B598, 8(2004).

14. A. Sibirtsev, J. Haidenbauer, S. Krewald, M. G. Meissner, A. W. Thomas, Phys. Rev. D 71, 054010 (2005).

15. For review, C. S. Gao and S. L. Zhu, Commun. Theor. Phys. 42, 844(2004); E. Klempt and A. Zaitsev, Phys. Rept. 454, 1 (2007).

16. K. Abe et al. (BELLE Collaboration), Phys. Rev. Lett. 94, 182002 (2005).

17. B. Aubert et al. (BABAR Collaboration), Phys. Rev. D 74,051101 (2006).

18. M. Ablikim et al. (BES Collaboration), Phys. Rev. Lett. 95, 262001 (2005).

19. M. Ablikim et al. (BESIII Collaboration), arXiv: 1012.3510, [hep-ex](2010).

20. M. Ablikim et al. (BESIII Collaboration), arXiv: 1012.5131, [hep-ex] (2010).

21. C. Hanhart, B. Kubis and J. R. Peláz, Phys. Rev. D 76,074028 (2007).

22. J. J. Wu, Q. Zhao and B. S. Zou, Phys. Rev. D 75, 114012, (2007).

23. J.J. Wu and B. S. Zou, Phys. Rev. D 78, 074017(2008).

24. M. Ablikim et al. (BESIII Collaboration), arXiv:1012.1117, [hep-ex](2010).

25. V. Dorofeev et al., Phys. Lett. B 651, 22 (2007).

26. R. A. Briere et al. (CLEO Collaboration), Phys. Rev. Lett. 84, 26(2000).

27. B. Borosoy and R. Nissler, Eur. Phys. J A 26, 383 (2005).

28. D. V. Amelin et al. , Phys. Atom. Nucl. 68, 373 (2005). 